\title{
Fragmentierende Stadtentwicklung unter Bedingungen der Globalisierung - Ergebnisse aus Santiago de Chile
}

\author{
Axel Borsdorf, Innsbruck, Rodrigo Hidalgo, Santiago \\ de Chile
}

\section{Einleitung}

Die seit circa 1980 fortschreitende Globalisierung hat nicht nur die internationalen Märkte und Wirtschaftsbeziehungen, die Struktur und Aktionsräume der ökonomischen Akteure, die politischen Rahmenbedingungen, sondern auch die Raumstrukturen massgeblich verändert. $\mathrm{Da}$ es sich um globale Prozesse handelt, sind im Prinzip alle Regionen der Erde betroffen, freilich in recht unterschiedlicher Weise. Diese Entwicklungen sind auch noch keineswegs zum Stillstand gekommen. Das Globalisierungsphänomen beinhaltet den freien Waren- und Kapitalverkehr und die Ausbildung internationaler Finanzmärkte unter neoliberaler Wirtschaftsdoktrin mit globalem Wettbewerb, die Informations- und Kommunikationsflüsse, den Bildungssektor, den Bedeutungsschwund nationaler Grenzen (WAGNLEITNER 2003), aber auch die Ausbreitung globaler Kultur- und Konsumformen (Mode, Musik, Nahrung, Lebensstile) und die internationale Dimension des Waren- und Personentransports inklusive der Mobilität von Personen, auch in multilokaler Form. Der Prozess ist durchaus heterogen und in sich widersprüchlich. Im Folgenden wird vor allem auf die wirtschaftliche und soziale Dimension fokussiert. Dabei führt die Globalisierung zur Verschärfung ethnisch-religiös-kultureller und sozialer Segregation und zur sozial- und wirtschaftsräumlichen Fragmentierung.

In seiner Weltsystemtheorie unterscheidet WALLERSTEIN (1974ff.) Weltreiche und Weltökonomie. Die gegenwärtige Globalisierung gehört zur zweiten Kategorie. Sie wird zwar von einzelnen Staaten stark vorangetrieben, global players sind vor allem aber transnationale Konzerne und Institutionen. Er betont wie auch Hirsch (1995) sowie Altvater und MAHNKopf (1996) die ökonomische Dimension, im Unterschied zu BECK (1997) und GidDENs (1990), die auch die ökologische, kulturelle, politische und zivilgesellschaftliche Dimension einbeziehen. Eine weitergehende Diskussion zur Wirkung der Globalisierung auf lateinamerikanische Städte bieten BorsDORF und CoY (2009), RoBERTs (2005) oder, in globaler Dimension, Lo und Yeung (1998) sowie SASSEN (2000).
Die Bewertungen dieser Prozesse unterscheiden sich. Wirtschaftlich und politisch wird die Globalisierung als Katalysator für Demokratisierung und Liberalisierung, als Wirtschaftsmotor und Instrument zur Hebung des allgemeinen Wohlstandes begrüsst, aber auch als Ursache der Verarmung, Instrument der Schaffung von Abhängigkeiten, Triebfeder der sozialräumlichen Segregation und Exklusion angesehen. Globalisierungsbefürworter und -gegner stehen sich diametral gegenüber. In diese Debatte soll mit dem vorliegenden Artikel aber nicht eingegriffen werden.

Vielmehr sollen bislang in dieser Diskussion wenig beachtete Phänomene dargestellt und diskutiert werden, die die veränderte Raumstruktur in von der Globalisierung erfassten Stadträumen Lateinamerikas betreffen. Studien hierzu wurden seit mehreren Jahren von den Verfassern und ihren Mitarbeitern und Kollegen in den Städten Mexiko (KANITSCHEIDer 2002; PARnreiter 2007), Quito (Kohler 2002) und Santiago de Chile durchgeführt. In diesem Beitrag sollen in erster Linie die Ergebnisse der langjährigen Untersuchungen in Santiago de Chile zusammengefasst werden. Dabei werden zunächst die raumstrukturellen Veränderungen behandelt, die sich unter dem Stichwort «Fragmentierung der Stadtstruktur» fassen lassen. In einem zweiten Teil stehen die Bevölkerungsbewegungen im Vordergrund. Sie belegen, dass die internationale Mobilität sehr wohl zur sozialen Enklavenbildung im Stadtzentrum beiträgt, während andererseits eine extreme Suburbanisierung in Chile nicht zur Postsuburbanisierung führte, sondern eher Charakteristika der Exurbanisierung aufweist.

Die Methode dieses Beitrags ist eine kompilatorische. Es werden die Ergebnisse von Teilstudien zu einer integrativen und in dieser Weise neuen Perspektive aufeinander bezogen und zusammengefasst. Die zahlreichen Karten und Tabellen, die die empirischen Ergebnisse zusammenfassen, werden in dieser Arbeit nicht noch einmal wiedergegeben, daher sind alle Abbildungen dieses Beitrags bislang unpubliziert. Auf die Zitation der Originalarbeiten der Verfasser, die die Empirie enthalten, wurde verzichtet, sie können aus der Projekthomepage (http:/www.uibk.ac.at/igf/ projekte/urbanisierung.html.de) entnommen werden. Die in den jeweils zugrundeliegenden empirischen Arbeitsphasen angewandten Methoden sind in anderen Beiträgen der Autoren z.T. ausführlich beschrieben. 
Der Erkenntniswert dieser Arbeit liegt in der Synthese. Es wird dabei versucht, die empirisch-analytischen Ergebnisse aus den Einzelprojekten in den theoretischen Rahmen des Modells der fragmentierenden Raumentwicklung zu integrieren, auch um die Leistungsfähigkeit dieser Theorie an einem Beispiel zu überprüfen.

\section{Die Umstrukturierung des Raumes als Folge der Globalisierung: das Modell der fragmentierenden Raumentwicklung}

Seinem Modell der fragmentierenden Raumentwicklung legt Scholz (2002) die Basisannahme zugrunde, dass Globalisierung der auslösende Faktor der Fragmentierung ist. Sie geht vor allem auf die neoliberale Wirtschaftspolitik zurück und hat eine «zweite Moderne» eingeleitet, deren Ausdruck der divergierende und fragmentierte Raum ist. Koop und ScHOLz (2001) haben diese Theorie am Beispiel von Mauritius und Pannhausen (2008) in Dar es Salaam empirisch überprüft.

Es darf nicht übersehen werden, dass die Globalisierung Menschen und Räumen viele Möglichkeiten bietet: Individuen und Regionen haben bessere Chancen auf Wohlstand, die geringere Regulation öffnet mehr Menschen Wege, sich in den Wirtschaftsprozess auch aktiv einzubringen, dabei eröffnen sich neue Chancen für den sozialen Aufstieg. Auch Standorte werden neu bewertet.

Die Globalisierung und die durch sie verursachte zweite Moderne führen aber auch zu einem Machtverlust der Staaten und zur teilweisen Auflösung der trennenden Kraft von Grenzen. Paradoxerweise gehen diese Bedeutungsverluste einher mit einer Zunahme der Bedeutung von Regionalismen: In Afrika gewinnen die ethnischen Territorien eine neue Bedeutung, ähnliche Prozesse sind in Mittelamerika zu beobachten. Regionalismen - als Antwort auf globale Homogenisierungstendenzen - nehmen zu, auch in Europa Dabei wachsen nicht nur die regionalen, sondern auch die sozialen Disparitäten. Im kleinräumigen Massstab nehmen die Abschottungsmechanismen der sozialen Schichten zu, es entstehen gated communities oder gar gated cities (MEYER \& BÄHR 2001). Mitverantwortlich für die Verstärkung der sozialräumlichen Exklusionstendenzen ist die verstärkte internationale Migration (Gómez 2008).

Wenn die Globalisierung auch Chancengleichheit verspricht, so haben doch ihre Akteure aufgrund ungleicher Verteilung von Ressourcen und Möglichkeiten unterschiedliche Handlungsoptionen. Grösste Macht haben die Kommandozentralen transnationaler Un- ternehmen und die in der Forschung und Entwicklung führenden technologischen Innovationszentren, unter Umständen auch fordistische Industrieorte. Sie bilden globale Orte als die höchste Hierarchiestufe. Globale Orte in diesem Verständnis sind nicht unbedingt Weltstädte, sondern Standorte dieser Unternehmen. Sie können in einer Stadt mit globalisierten Orten und der marginalisierten Restwelt koexistieren.

Auf der zweiten Ebene befinden sich die globalisierten Akteure in globalisierten Orten. Dies sind unternehmensorientierte Dienstleistungszentren, offshoreBankenzentren, aber auch Standorte verlängerter Werkbänke und der industriellen Massenproduktion, Zentren, die Bergbauprodukte oder Agrarerzeugnisse für den Weltmarkt liefern, oder internationale Tourismusdestinationen. Santiago de Chile, aber auch Mexiko-Stadt oder Quito gehören zu diesen von der Globalisierung betroffenen Städten, sie sind jedoch keine echten global players, weil die dort ansässigen Firmenzentralen nur Regionalzentren transnationaler Unternehmen sind oder aber nur einen regionalen Aktionsraum (Mittelamerika, Andenraum, Lateinamerika) haben. Häufig sind solche Standorte durch einen hohen Anteil des informellen Sektors gekennzeichnet. Dieser informelle Sektor charakterisiert die dritte Hierarchiestufe des globalisierten Systems, die neue Peripherie oder das Meer der Armut. Dort vereinen sich die Globalisierungsverlierer.

Das hier beschriebene System ist nicht starr, sondern ausserordentlich dynamisch. Dafür ist der ständige Wettbewerb verantwortlich, der in jedem Moment zum Sturz aus einer Stufe auf eine andere oder aber zum (freien) Fall führen kann. Diese Dynamik ermöglicht jedoch ebenso einen raschen Aufstieg aus der Peripherie in die globalisierte Welt oder gar die Stufe der global players. Ein weiteres Charakteristikum dieses Systems ist, dass es nicht nur auf Personen und Institutionen bezogen ist, sondern auch auf Räume. Auch diese können an Bedeutung gewinnen und zu global cities aufsteigen, ebenso wie sie einen plötzlichen Bedeutungsverlust erleiden können.

Mit dem Modell der räumlich fragmentierenden Entwicklung wird dieses System auf einer räumlichen Ebene dargestellt. Es muss betont werden, dass Fragmentierung jedoch kein rein raumstrukturelles Phänomen ist, sondern auch die Sozialstruktur (z.B. Fragmente nach Einkommen, Schicht, Bildung, Ethnien) umfasst.

Der fragmentierte Raum ist ein aus einer Grundmatrix (Meer der Armen) und darin eingelagerten Fragmenten (Inseln) der globalisierten oder der globalen Ebene bestehendes System. Es muss nicht notwendigerweise an Städte gebunden sein, obwohl dies 
der Normalfall ist. Deshalb kann im Folgenden auch von fragmentierender Stadtentwicklung gesprochen werden.

Hierfür hat HaLl (1998) ein Modell entworfen, das in gewisser Weise mit der Theorie von Scholz korrespondiert und von anderen Autoren weiterentwickelt wurde (vgl. SASSEN 2000). In dieser global city sind die Kommandozentralen der global players im Zentrum lokalisiert, das freilich durch polyzentrische Strukturen ergänzt wird. An der Peripherie befinden sich die gated communities und cities der Reichen sowie mit den edge cities auch schon postsuburbane Strukturen. Es wird nun zu überprüfen sein, ob und inwiefern die von Scholz (2002) und Hall (1998) postulierten Raumstrukturen sich auch in der globalisierten Agglomeration Santiago de Chile wiederfinden.

\section{Die Fragmentierung des städtischen Raumes in lateinamerikanischen Hauptstädten: das Beispiel Santiago de Chile}

Die chilenische Hauptstadt Santiago eignet sich in besonderer Weise zur Darstellung der Effekte der Globalisierung in der urbanen Struktur, weil dort die Globalisierung besonders abrupt und sehr früh einsetzte, und zwar circa ein Jahr nach der Machtübernahme durch die Militärjunta unter General PINOCHET (DE MAtTos 2003). Nach anfänglicher Unschlüssigkeit setzte diese auf den Weltmarkt, so dass Chile bald als Musterland des Neoliberalismus und Vorreiter der Globalisierung galt. Tatsächlich führten die Massnahmen der Militärregierung auch sehr rasch zu einer Konsolidierung der Nationalökonomie Chiles, die freilich mit hohen sozialen Kosten erkauft wurde. Da die Regierung über 17 Jahre an der Macht blieb und sich - von zwei Plebisziten abgesehen - keiner Wahl zu stellen hatte, war die Umstellung der Wirtschaftspolitik dauerhaft und wurde, wegen des prinzipiellen Erfolges, auch von den demokratischen Folgeregierungen nicht geändert. Chile ist somit das Land des Südens mit der längsten neoliberalen Tradition.

\subsection{Sozialräumliche Segregation auf unterschiedlichen Massstabsebenen}

Sabatini (1998) sowie Cáceres und SABatini (2004) haben auf die unterschiedlichen Massstabsebenen der sozialräumlichen Segregation in Santiago de Chile hingewiesen. Räumliche Exklusion findet diesen Autoren zufolge zugleich auf einer grossräumigen Ebene (reiche versus arme Stadt) und auf einer kleinräumigen Ebene (Abkapselung von barrios cerrados) statt.

Für Mexiko-Stadt zeigten Kanitscheider (2002) und KoHLer (2005), dass ein solches Bild immer noch zu kurz greift. Sie konnten die Ergebnisse von SABATINI (1998) bestätigen, aber auch aufzeigen, dass sich die Segregation vielfach auch auf die manzana-Ebene erstreckt (Wohnblockebene). Demnach können sich in einer einzigen manzana abgeschottete «reiche» Wohneinheiten neben armen befinden.

Ähnliche Ergebnisse haben die Autoren auch für Santiago de Chile gefunden und damit die Resultate von SABATINI modifiziert. Sie konnten darüber hinaus auch nachweisen, dass die Disparität von armer und reicher Stadt eine Altstruktur ist, die heute zunehmend durch diffizilere kleinräumigere Segregationsformen modifiziert oder gar aufgelöst wird.

Demnach gibt es neben der grossräumigen Teilung der Stadt in eine «reiche» und eine «arme» Stadt (Makrosegregation) auf einer kleinräumigen Massstabsebene auch eine weit differenziertere Segregation. Im «Meer der Armen», also durchaus auch in der «armen Stadt», finden sich durch hohe Mauern abgeschottete barrios cerrados (gated communities) der Ober- und Mittelschicht, umgekehrt sind aber auch in der reichen Stadt Inseln der Armen zu finden.

Die mehrdimensionale Struktur der Segregation kann als Makro-, Meso- und Mikrosegregation bezeichnet werden. Auf der Makroebene ist dies die alte Polarisierung in eine arme und eine reiche Stadt. Der Mesoebene sind alle grossflächigen barrios cerrados zuzuordnen, also vor allem jene, die aus Bungalows bestehen. Dazu zählen auch die «Neuen Städte» an der Peripherie, die im Endausbau für 50.000 Einwohner und mehr geplant, nur für eine Sozialschicht vorgesehen und gänzlich von der Umgebung abgesperrt sind. Mikrosegregation liegt dann vor, wenn im Wohnblock einzelne Komplexe (Wohntürme, kleinere Reihenhauseinheiten) sozial einheitlich bewohnt und mit Sicherheitseinrichtungen versehen sind und in derselben manzana auch Grundstücke von anderen Sozialschichten bewohnt werden.

In der globalisierten Stadt spiegeln sich daher die Prozesse einer Fraktalisierung (FrankHAUser 2002). Sie sind Kennzeichen globalisierter Stadtentwicklung (Abb. 1).

Sichtbares Zeichen der akzentuierten Segregation sind die Sicherheitsanlagen, die die für eine Sozialschicht vorbehaltenen Wohnkomplexe absichern. Dies sind immer Mauern und Zäune sowie zentrale Eingangstore, hinzu kommen oft Wachttürme, 24-Stunden-Sicherheitspersonal, Videoüberwachung, Passkontrolle und andere Systeme. Derart abgeschottete Siedlungen werden barrios cerrados genannt. Sie sind rechtlich Gemeinschaftseigentum (daher auch der Name condominio). Die Eigentümer erwerben einen 


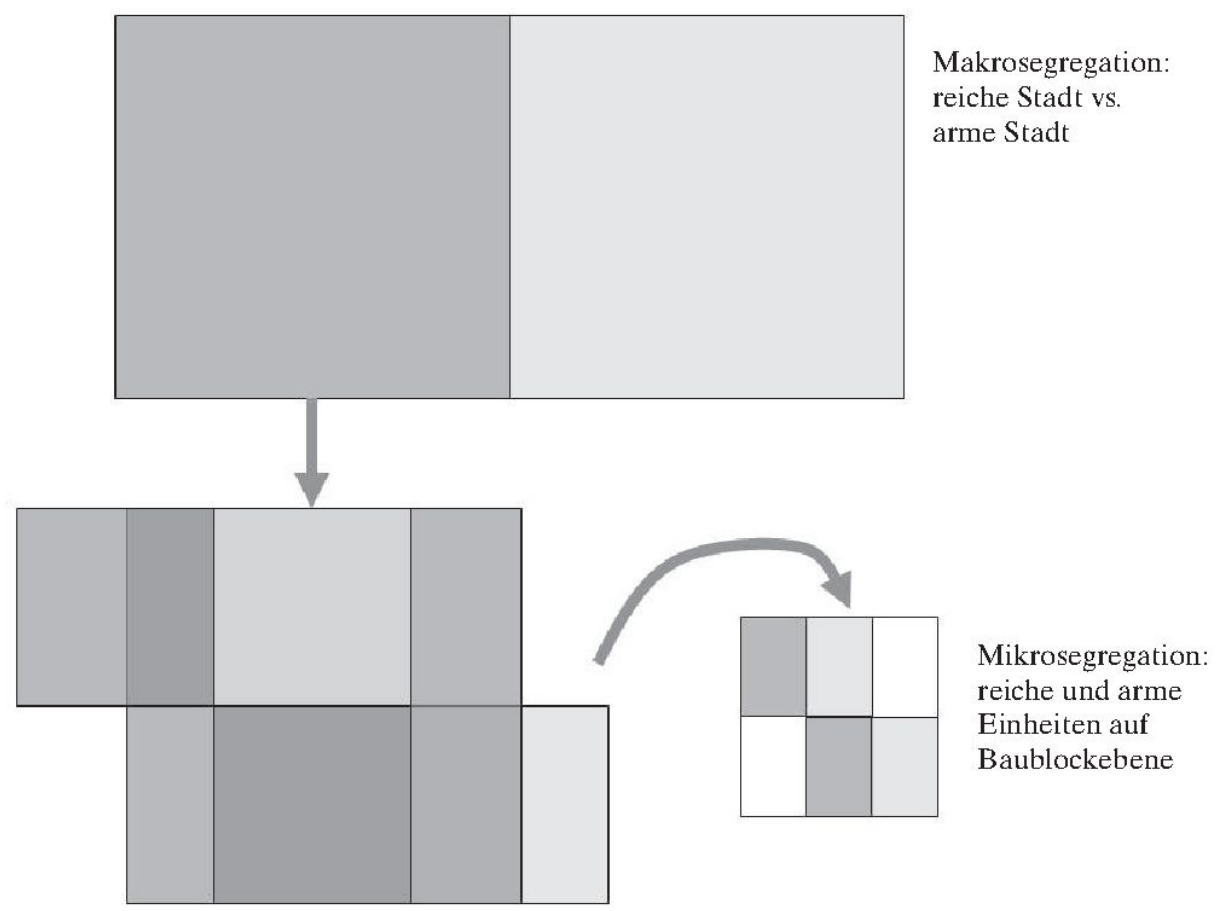

Mesosegregation:

grosse barrios cerrados und ciudades valladas

Abb. 1: Fraktale in der fragmentierten Stadt

Fractals in the fragmented city

Fractales dans la ville fragmentée

Entwurf: A. BoRsDoRF

Anteil des Gesamtgrundstücks, besitzen aber ihre Wohnung bzw. ihr Haus. Es können horizontale (Bungalowviertel) und vertikale condominios (Appartementhochhäuser) unterschieden werden. Die «Condominisierung» (PLöGER 2006) greift inzwischen auf die traditionellen Stadtviertel über, wo Strassenzüge abgesperrt werden.

Mit der Ummauerung wird es möglich, dass Viertel der Ober- und Mittelschicht in ehemals als arm geltender Umgebung angelegt werden (GALlEguillos ArAYASCHÜBELIN 2007). Die Nachbarschaft von Reich und Arm wird von den wohlhabenden Bürgern als durchaus positiv gesehen, weil sie für ihre Grundstücke und Häuser Personal benötigen, das in enger räumlicher Nähe rekrutiert werden kann.

Daher verteilen sich die barrios cerrados zunehmend im ganzen Stadtgebiet. Zwar ist eine gewisse Dominanz in den traditionellen Vierteln der Ober- und Mittelschicht im Westen zu erkennen, aber die abgeschotteten Viertel finden sich heute über die ganze Stadt verteilt. Die Abschottung von Wohneinheiten hat freilich in Lateinamerika eine lange Tradition. Insofern traf die mit der Globalisierung verbundene Privatisierung öffentlichen Raums in Chile auf eine dafür aufnahmefähige Bevölkerung.

\subsection{Polyzentrische Strukturen}

Während BÄHR und MERTINs (1995) noch davon ausgingen, dass moderne malls und Einkaufszentren als Motoren und neue Zentren der Stadtentwicklung in den reichen Vierteln der lateinamerikanischen Grossstadt wirken, haben verschiedene Arbeiten und Kartierungen der Autoren gezeigt, dass solche Einrichtungen, die aufgrund ihres multifunktionalen Charakters als urban entertainment centres bezeichnet werden können, sich im gesamten Stadtgebiet verteilen. Die Konsumtempel wurden dabei nach malls (mit mehreren Ankergeschäften und zentralen plazas mit Unterhaltungsangebot), Einkaufszentren (ohne plaza und meist mit nur einem oder keinem Ankergeschäft), Hypermärkten (mit einem grossen Ankergeschäft und wenigen kleinen Fachgeschäften) und Fachmarktagglomerationen (mit mehreren nicht integrierten Fachmärkten) klassifiziert. Dabei zeigt sich, dass sie 


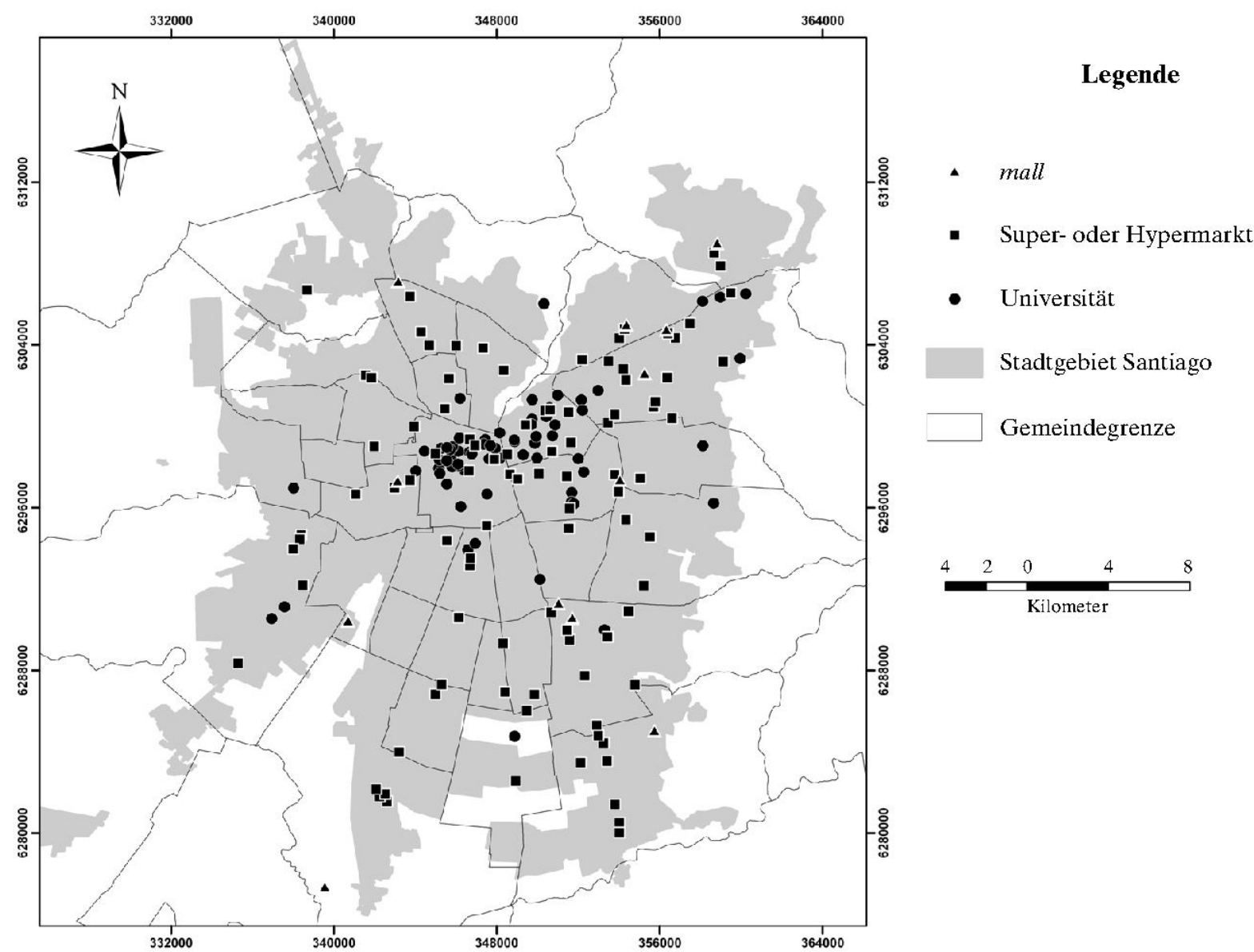

Abb. 2: Malls, Märkte und Universitäten in Santiago 2008

Malls, markets and universities in Santiago in 2008

Centres commerciaux, commerces et universités à Santiago, 2008

Quelle: eigene Kartierung

sich in der ehemals «reichen» Stadt konzentrieren, in jüngster Zeit aber auch in der ehemals «armen» Stadt immer mehr derartige Konsumtempel entstehen.

In gleicher Weise kennzeichnend für die Ausprägung der fragmentierten Struktur sind die neuen, meist von transnationalen Investoren errichteten Industrieparks und business centres. Sie werden an Schnittpunkten der Hauptverkehrsachsen angelegt, schotten sich aber auf gleiche Weise ab wie die gated communities. Sie lösen mehr und mehr die alten Industriegassen ab, die sich an den alten Ausfallstrassen bzw. Eisenbahnlinien gebildet hatten.

Auch im Dienstleistungssektor gibt es ähnliche Raumstrukturen. Die bis 1970 noch weitgehend durch Wohnbauten gekennzeichnete Oberstadt hat inzwischen zahlreiche Bürohochhäuser erhalten, die sich teils clusterartig verdichten, teils aber auch inselhaft in Wohngebieten errichtet werden. Jeder Turm ist für sich abgeschottet und mit einem eigenen Sicherheitssystem versehen, durchaus ähnlich zu den benachbarten Appartementtürmen, die mit ihren Sicherheitssystemen auch als vertikale barrios cerrados angesehen werden. Büro- wie auch Wohnblocks werden oft von transnational agierenden Immobiliengesellschaften errichtet.

Schliesslich verstärken auch die noch immer anhaltenden Gründungen von Privatuniversitäten die Fragmentierung des Stadtraumes, indem sie sich über das Stadtgebiet verteilen (Abb. 2). Auch diese werden - von Ausnahmen abgesehen - von international im Bildungssektor tätigen Unternehmen errichtet und betrieben.

\subsection{Neue Formen der Suburbanisierung}

Als die chilenische Regierung 1980 das Decreto 3.516 zur Unteilbarkeit landwirtschaftlicher Grundstücke 
ab $5000 \mathrm{~m}^{2}$ Grösse erliess, sah der Immobiliensektor rasch seine Chance. Firmen kauften grössere landwirtschaftliche Flächen und teilten diese in $5000 \mathrm{~m}^{2}$ grosse Einheiten auf. Diese Grundstücke wurden als parcelas de agrado ausgewiesen und an reiche Interessenten zur Überbauung mit Luxusvillen verkauft, wobei die Gesamtfläche des einstigen Grundstücks eingezäunt und gesichert wurde. Die Käufer der parcelas werden zwar - anders als die Käufer von Einheiten in einem condominio - wirkliche Eigentümer (mit Grundbucheintragung), wegen der Einfriedung und Sicherung solcher aus parcelas bestehenden Einheiten können diese aber auch als de facto condominios (in Unterschied zu ex lege condominios) bezeichnet werden.

In Santiago sind solche Komplexe vor allem in den südlichen und westlichen Gemeinden sowie ausserhalb der Metropolitanregion zu finden. Zwischen 1994 und 2002 entstanden insgesamt 156.251 parcelas in circa 7500 abgezäunten Anlagen.

Seit Jahrzehnten liegt das Wachstum von Santiago de Chile (1992-2002: 8,6\%) - wie das vieler anderer lateinamerikanischer Megastädte - unter der mittleren Regenerationsrate des Landes (1992-2002: $12,8 \%$ ). Dennoch wächst die Stadt weiter stürmisch in die Fläche, da das Bedürfnis der urbanen Bevölkerung nach grösserem Wohnraum noch lange nicht gestillt ist, wobei die Wachstumsrate der Stadtfläche in der letzten Dekade etwa doppelt so hoch war wie die der Bevölkerung. Man kann daher fragen, ob der Stadt die Fläche ausgehen wird. Das Wachstum konzentriert sich auf die periurbanen Gemeinden, die ihre Einwohnerzahl in der letzten Dekade zum Teil verdoppeln konnten, während nahezu alle zentralen Gemeinden Bevölkerung verloren, und zwar bis zu 16\%. Die Gemeinden Colina und Lampa verzeichneten zwischen 1992 und 2002 einen Bevölkerungszuwachs um $47,1 \%$ bzw. $60,2 \%$ (DE MatTos 2003 ).

Diese grossen Wachstumsraten sind auf die Anlage von neuen Städten seit den 1990er Jahren zurückzuführen. Es sind dies neue Städte ab 50.000 bis über 100.000 Einwohner, die mit Mauern, Zäunen, Toren und Sicherheitssystemen hermetisch von der Aussenwelt abgeschlossen und mit eigenen Versorgungs- und Dienstleistungsfunktionen versehen, aber nur je einer bestimmten Sozialschicht vorbehalten sind. Sie werden ciudades valladas (eingezäunte Städte) genannt und sind im Übrigen nicht auf Santiago beschränkt, sondern auch aus Brasilien (CoY \& PöHLER 2002) und Argentinien (JANOSCHKA 2002) bekannt.

Parcelas de agrado sind ebenso wie die neuen ciudades valladas als Periurbanisierungsphänomene $\mathrm{zu}$ betrachten. Sie entleeren sich tagsüber und füllen sich am Abend. Dennoch sind sie auch Ausdruck der Frag- mentierung des Stadtorganismus, da beide Formen zellenhaft und insular als in sich einheitliche Fragmente entstehen. Sie widersprechen somit nicht dem Modell der fragmentierenden Entwicklung. Dennoch muss auch gesagt werden, dass in Santiago das marginalisierte Gebiet einen wesentlich kleineren Raum einnimmt als dies ScHolz in seinem Modell annimmt (vgl. Abb. 3).

\section{Neue Bevölkerungsbewegungen als Folge der Globalisierung}

\subsection{Amenity migration}

Die Oberschicht nimmt in den letzten Jahren immer mehr die Möglichkeit wahr, Zweithäuser und -wohnungen, aber auch Hauptwohnsitze aufgrund der ökologischen und kulturellen Vorzüge (=amenities) in grosser Entfernung von der Agglomeration zu beziehen. Diese Stadtflucht führt aber nicht zu post-suburbanen Strukturen, sie ist vielmehr ein Phänomen der amenity migration, wie sie vor allem aus den Vereinigten Staaten, aber auch aus Europa bekannt ist (Moss 2006). In Santiago sind es vor allem die Küstenkordillere und der Cajón del Maipo, ein Hochtal der Hochkordillere, in denen auf grosszügigen Grundstücken, den parcelas de agrado, mit Schwimmbecken ausgestattete, individuell gestaltete Villen erbaut werden. Die Küstenkordillere ist auch für die reichen Schichten von Valparaíso/Viña del Mar attraktiv.

Der Ferienort Olmué, rund 80 km von der Hauptstadt entfernt, ist ein Beispiel dieser Entwicklung. Pullfaktoren sind die gute Luft, das als besonders gesund geltende Mikroklima, die schöne Landschaft, die Nähe eines Nationalparks und Biosphärenreservats, die lebendige ländliche Kultur mit rodeos und anderen amenities. Diese Entwicklung wurde aber erst durch die Einrichtung einer permanenten Buslinie mit komfortablen Pullman-Fahrzeugen und der Asphaltierung der mautfreien Zugangsstrasse ermöglicht, die die Fahrtzeit von zwei auf eine Stunde verkürzte.

Die amenities des Kordillerentals Cajón del Maipo sind noch attraktiver als die von Olmué. Die Berge sind höher, die Skizentren näher, der Fluss erlaubt Rafting-Abenteuer, die touristische Infrastruktur ist älter und «authentischer», die Kultur autochthoner. Naturparks laden zum Wandern, heisse Quellen zur Wellness ein. Die Entfernung zum Stadtzentrum ist mit rund $50 \mathrm{~km}$ und unter einer Stunde Fahrtzeit geringer als im Fall von Olmué.

Die Abwanderung von Mitgliedern der Oberschicht, die auf amenity-Faktoren der Zielorte beruht, hat inzwischen ein Ausmass erreicht, das zwar in Relation zur Gesamtbevölkerung gering ist, räumlich jedoch 


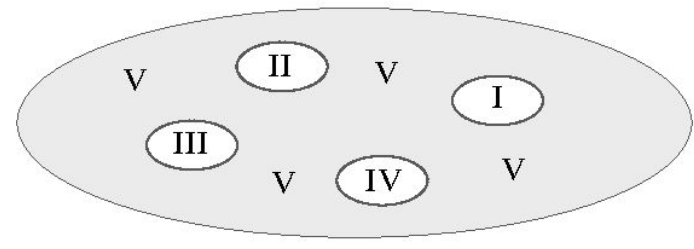

Fragmente (nach Scholz 2002):

I Industrie

II barrios cerrados

III Bürotürme und -cluster

IV Entertainmentcenter, malls, Clubs

Neue Peripherie

V Marginalviertel

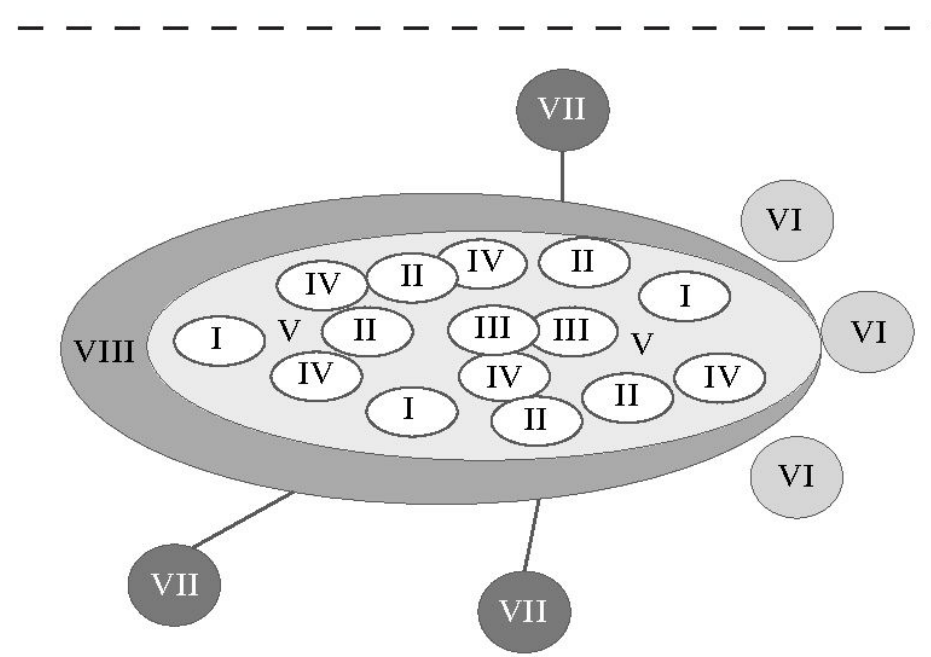

Fragmente in Santiago:

I Industrie

II barrios cerrados

III Bürotürme und -cluster

IV Entertainmentcenter, malls, Clubs

V Marginalviertel

VI Neue Städte (ciudades valladas)

VII amenity migration-Zielorte

VIII Sozialer Wohnungsbau

\begin{abstract}
Abb. 3: Vergleich des Modells der fragmentierenden Stadtentwicklung mit der Situation in Santiago de Chile Comparison of the model of fragmented urban development and the situation in Santiago de Chile Comparaison entre le modèle du développement urbain fragmenté et la situation à Santiago du Chili Quelle: nach ScHoLz 2002, modifiziert
\end{abstract}

grosse Wirkung hat. Es kann geschätzt werden, dass zwischen 1991 und 2002 circa 1200 Personen in die untersuchten Zielgebiete Olmué, Curacaví und Cajón del Maipo abgewandert sind. Dort waren bis 2002 insgesamt 337 parcelas de agrado mit einer Gesamtfläche von 17,3 ha entstanden.

Die amenity migration ist in Chile neu, begünstigende Faktoren sind der Rückzug des Staates aus der Raumordnung, die Deregulierung, die Privatisierung des Bodenmarktes und die zunehmende Mobilität. Sie ist ein konstitutives Element der Fragmentierung des Raumes. Insofern bestätigt sie die Thesen von Scholz (2002). Die Beispiele belegen aber auch, dass es dabei noch nicht zu den aus den USA bekannten Phänomenen der Exurbanisierung und zur edge-cityBildung gekommen ist. Insofern fehlen also in der chilenischen Entwicklung noch Phänomene, die Hall (1998) in sein Modell der Global City aufgenommen hat.

Abb. 3 fasst die bisherigen Ergebnisse zusammen und vergleicht sie mit dem Modell der fragmentierenden Stadtentwicklung von ScHoLz (2002).

\subsection{Internationale Einwanderung}

Die Öffnung der Märkte, die Vereinheitlichung von Arbeitsmärkten, der Abbau von Grenzformalitäten und die Senkung von Transportkosten begünstigten die internationale Mobilität, die daher auch als ein Effekt der Globalisierung angesehen wird. Chile - ursprünglich ein Einwanderungsland wurde 1970 zum Auswanderungsland, als Gegner der sozialistischen Regierung in grossen Scharen das Land verliessen. Diese Tendenz verstärkte sich nach dem Militärputsch, als zahlreiche Regimegegner flüchteten oder des Landes verwiesen wurden. Seit 1990 lässt sich beobachten, dass Chile wieder zum Einwanderungsland geworden ist, und zwar als Wunschdestination von Menschen aus Nachbarländern.

Das Wohlstandsgefälle hat zur Immigration von Argentiniern geführt, die in Chile meist gut bezahlte Arbeitsplätze finden und im öffentlichen Leben als solche kaum auffallen. Anders verhält es sich mit der Zuwanderung von Peruanern. Sie sind nach Hautfarbe «sichtbar», üben in grossem Umfang Tätigkeiten im informellen Sektor aus, der zuvor in Chile auf ein 
geringes Ausmass geschrumpft war, und sie konzentrieren diese Tätigkeiten räumlich im alten Zentrum von Santiago, wo sie als «Menge» auch wahrgenommen werden. Obwohl die argentinischen Einwanderer $26 \%$ der gesamten Zuwanderung der letzten Dekade ausmachten und die Peruaner nur $21 \%$ dazu beitrugen, regt sich in der Öffentlichkeit nur Widerstand gegen diese peruanischen Migranten, der in der Presse, im Rundfunk und Fernsehen, aber auch bei den politischen Parteien einen nachhaltigen Widerhall findet (Gómez 2008).

Nicht nur in Chile gibt es neben der offiziellen Einwanderung auch eine inoffizielle. Wurden in der letzten Volkszählung 38.000 peruanische Zuwanderer registriert, geht das chilenische Aussenministerium von einer faktischen Migration aus Peru im Jahr 2001 von 81.000 Personen aus. Dies ist im internationalen Vergleich gering.

Die männlichen Peruaner wohnen vor allem im Stadtzentrum, wo sie auch arbeiten. Die weiblichen Einwanderer dagegen sind überwiegend als Hausmädchen in den Haushalten der Oberschicht tätig, wo sie auch wohnen. Sie sind für die Konzentration von Peruanern im Barrio Alto von Las Condes verantwortlich.

Die Konzentration der Peruaner im Stadtzentrum belegt die These von ScHolz (2002), dass die Fragmentierung auch die Sozialstruktur umfasst und ist stimmig zu seinem Modell der fragmentierenden Entwicklung.

\section{Fazit}

Die mehr als fünfjährigen Arbeiten der Verfasser und ihrer Forschungsteams aus Chile und Österreich haben dokumentiert, dass Santiago de Chile unter Einfluss der nun seit 35 Jahren anhaltenden Globalisierung einen Strukturwandel zur «globalisierten» Stadt durchgemacht hat. In Bezug auf die beschleunigte Segregation auf drei Massstabsebenen, die Entwicklung polyzentrischer Strukturen, die ethnische, soziale und funktionale Fragmentierung und die Einleitung neuer Migrationen in die Peripherie entspricht die Entwicklung von Santiago weitgehend der ScHolzschenTheorie.

Im Modell der lateinamerikanischen Stadtentwicklung (BoRsDORF, BÄHR \& JANOSCHKA 2002) wird diese Fragmentierung deutlich. Sie löst die früheren Strukturmuster der kompakten (kolonialzeitlichen), der sektoralen (nach der ersten Urbanisierungsphase) und bipolaren Stadt (am Ende der zweiten Urbanisierungsphase) ab. Dies haben auch BÄHR und MEYERKRIESTEN (2007) nachgewiesen.
Nur dort, wo der Staat noch führend ist (Verkehrsinfrastruktur, öffentliche Dienstleistungen, Sozialer Wohnungsbau), ist es bei der Orientierung an den traditionellen Raummustern der gravitativen und polarisierten Struktur geblieben. Der öffentliche Personennahverkehr ist nach wie vor sternförmig ausgerichtet, Ringlinien, die einen grossen Teil der Verkehrsströme aufnehmen könnten, bestehen nur für den motorisierten Individualverkehr.

Dort, wo die globalen Marktkräfte wirken, entstehen fragmentierte Strukturen; wo der Staat noch Einfluss hat, wird dagegen an den überkommenen Raummustern des Zentrum-Peripherie-Modells festgehalten.

Das von Hall (1998) entwickelte Modell der Global City trifft hingegen auf Santiago nur sehr bedingt zu. Obwohl es Exurbanisierungsphänomene (amenity migration) gibt, sind Postsuburbanisierungs- und Desurbanisierungstrends noch nicht festzustellen. Auch sind die Arbeiterviertel in Santiago keineswegs kreisförmig um den central business district herum angelegt. Andererseits entspricht die polyzentrische Struktur Santiagos dem Modell, ebenso wie die sozialräumliche Segregation in Form der barrios cerrados.

Santiago, wo die durch die neoliberale Doktrin verursachte (ökonomische und in der Folge auch soziale) Globalisierung schon sehr früh einsetzte, ist ein gutes Beispiel für die Effekte des Phänomens. Soziale und ethnische Segregation, die Privatisierung des öffentlichen Raums durch gated communities und cities, die Entstehung von sozialen und ökonomischen Fragmenten, internationale Mobilität sind Ergebnisse dieses Prozesses.

\section{Dank}

Die Autoren danken der Chilenischen Forschungsförderung Fondecyt für die stetige Unterstützung, zuletzt im Projekt $N^{\circ} 1095222$.

\section{Literatur}

Altvater, E. \& B. Mahnkopf (1996): Grenzen der Globalisierung. Ökonomie, Ökologie und Politik in der Weltgesellschaft. - Münster: Westfälisches Dampfboot.

BÄHR, J. \& G. Mertins (1995): Die lateinamerikanische Grossstadt. Verstädterungsprozesse und Stadtstrukturen. - Erträge der Forschung, Band 288, Darmstadt: Wissenschaftliche Buchgesellschaft.

BÄHr, J. \& K. Meyer-Kriesten (2007): Santiago de Chile - eine fragmentierte Stadt? Eine faktorenanalytische Untersuchung der Stadtstruktur in $2002 \mathrm{im}$ Vergleich zu 1970. - In: Erdkunde 61:258-276. 
BEck, U. (1997): Was ist Globalisierung? Irrtümer des Globalismus - Antworten auf Globalisierung. - Frankfurt am Main: Suhrkamp.

Borsdorf, A., BÄHr, J. \& M. JANOSCHKA (2002): Die Dynamik stadtstrukturellen Wandels in Lateinamerika im Modell der lateinamerikanischen Stadt. - In: Geographica Helvetica 57, 4: 300-310.

Borsdorf, A. \& M. CoY (2009): Megacities and global change: case studies from Latin America. - In: Die Erde 140, 4: 1-20.

Cáceres, G. \& F. Sabatini (Hrsg.) (2004): Barrios cerrados en Santiago de Chile: entre la exclusión y la integración residencial. - Santiago de Chile: Eurelibros, Instituto de Estudios Urbanos, Pontificia Universidad Católica de Chile.

CoY, M. \& M. PöHLER (2002): Condomínios fechados und die Fragmentierung der brasilianischen Stadt. Typen, Akteure, Folgewirkungen. - In: Geographica Helvetica 57, 4: 264-277.

De MatTos, C. (2003): Globalización y transformación metropolitana en el caso de Santiago. - In: Arenas, F., Hidalgo, R. \& J.L. Coll (Hrsg.): Los nuevos modos de gestión de la metropolización. - Santiago de Chile: Geolibros, Instituto de Geografia, Pontificia Universidad Católica de Chile: 27-55.

Frankhauser, P. (2002): La «ville fractale» et la fractalité des villes. - In: Borsdorf, A. \& C. PARnReITER (Hrsg.): International research on metropolises. Milestones and frontiers. - ISR-Forschungsberichte 29, Wien: Verlag der Österreichischen Akademie der Wissenschaften: 65-83.

Galleguillos Araya-Schübelin, M.X. (2007): Möglichkeiten zum Abbau von Segregation in Armenvierteln. Die Frage nach der sozialen und ökonomischen Nachhaltigkeit urbaner Ballungsräume am Beispiel Santiago de Chile. - Kieler geographische Schriften 115, Kiel: Geographisches Institut der Universität.

Giddens, A. (1990): The consequences of modernity.Stanford, California: Stanford University Press.

Gómez, A. (2008): Peruanische Immigration in Santiago de Chile. - Unveröffentlichte Diplomarbeit, Institut für Geographie und Regionalforschung der Universität Wien.

Hall, P.G. (1998): Cities in civilization. Culture, innovation and urban order. - London: Weidenfeld \& Nicolson.

Hirsch, J. (1995): Der nationale Wettbewerbsstaat. Staat, Demokratie und Politik im globalen Kapitalismus. - Berlin: Edition ID-Archiv.

JANoschKa, M. (2002): Wohlstand hinter Mauern. Private Urbanisierungen in Buenos Aires. - ISR Forschungsberichte 28, Wien: Verlag der Österreichischen Akademie der Wissenschaften.

Kanitscheider, S. (2002): Condominios und fraccionamientos cerrados in Mexiko-Stadt. Sozialräumliche
Segregation am Beispiel abgesperrter Wohnviertel. Geographica Helvetica 57, 4: 253-263.

KoHLer, P. (2002): Geschlossene Wohnviertel in Quito. Naturraum und rechtliche Rahmenbedingungen als Einflussgrössen für Verbreitung und Typisierung. - In: Geographica Helvetica 57, 4:278-289.

Koop, K. \& F. Scholz (2001): Mauritius - Beispiel für die Unmöglichkeit nachholender Entwicklung im Zeitalter der Globalisierung. - In: Nord-Süd aktuell 15, 3: 525-535.

Lo, F. \& Y. YEung (Hrsg.) (1998): Globalization and the world of large cities. - Tokio: United Nations University Press.

Meyer, K. \& J. BÄHr (2001): Condominios in Greater Santiago de Chile and their impact on the urban structure. - In: Die Erde 132, 3: 293-321.

Moss, L.A.G. (Hrsg.) (2006): The amenity migrants. Seeking and sustaining mountains and their cultures. - Wallingford: CABI.

Pannhausen, C. (2008): Städtische Landwirtschaft in Dar es Salaam: eine Untersuchung zur Theorie der fragmentierenden Entwicklung in einem globalisierten Ort. - Unveröffentlichte Diplomarbeit, Geographisches Institut, Universität Bonn.

Parnreiter, C. (2007): Historische Geographien, verräumlichte Geschichte. Mexico City und das mexikanische Städtenetz von der Industrialisierung bis zur Globalisierung. - Stuttgart: Franz Steiner Verlag.

Plöger, J. (2006): Die nachträglich abgeschotteten Nachbarschaften in Lima (Peru). Eine Analyse sozialräumlicher Kontrollmassnahmen im Kontext zunehmender Unsicherheiten. - Kieler geographische Schriften 112, Kiel: Geographisches Institut der Universität.

RoberTs, B.R. (2005): Globalization and Latin American cities. - In: International Journal of Urban and Regional Research 29, 3: 110-123.

Sabatini, F. (1998): Transformación urbana y dialéctica entre integración y exclusión. Reflexiones sobre las ciudades Latinoamericanas y notas sobre Santiago de Chile. - Santiago de Chile: Instituto de Estudios Urbanos, Pontificia Universidad Católica de Chile.

SAssen, S. (2000): Spatialities and temporalities of the global: elements for a theoretization. - In: Public Culture 12, 1: 215-232.

Scholz, F. (2002): Die Theorie der «fragmentierenden Entwicklung». - Geographische Rundschau 54, 10: 6-11.

Schumann, H. (1997): Die Globalisierungsfalle. http://www.gwdg.de/ fsbio/speech.htm 1.6.2011.

WAGnleitner, R. (2003): Was heisst Globalisierung? - In: Aurora, Magazin für Kultur, Wissen und Gesellschaft, http:/www.aurora-magazin.at/gesellschaft/ global_wagnleitner_gl_frm.htm 1.6.2011.

WALleRstein, I. (1974, 1980, 1989): The modern world system. - 3 Bände, New York: Academic Press. 


\begin{abstract}
Zusammenfassung: Fragmentierende Stadtentwicklung unter Bedingungen der Globalisierung - Ergebnisse aus Santiago de Chile

Die Globalisierung hat die städtische Struktur aller lateinamerikanischen Städte massgeblich verändert. Chile war das erste Land des Subkontinents, das neoliberale Reformen bereits in den 1970er Jahren durchgeführt hat. Seit dieser Zeit beeinflusst der Globale Wandel (Klimawandel und Globalisierung) vor allem die Hauptstadt Santiago de Chile. Die fragmentierende Entwicklung lässt sich seit dieser Zeit im Stadtorganismus beobachten. Der vorliegende Artikel fasst die Ergebnisse verschiedener Forschungsprojekte, die in den letzten Jahren von den Autoren durchgeführt wurden, zusammen. Sie belegen, dass Fragmentierung nicht nur zu einem neuen Muster sozialer und ethnischer Segregation und zur Neuverteilung der Einzelhandels- und Dienstleistungszentren führt, sondern auch neue Formen der Mobilität und Migration zur Folge hat.
\end{abstract}

Schlüsselwörter: Stadtentwicklung, Globalisierung, Chile, Segregation, amenity migration

\section{Summary: Fragmented urban development in the light of globalisation - results from research in Santiago de Chile}

Globalisation has significantly changed the face of urban space in Latin American cities. Chile was the first country on the subcontinent to initiate neoliberal reforms in 1970s. Since then, global change (climate change and globalisation) has clearly marked the capital city, Santiago de Chile, with signs of fragmented development everywhere in the urban fabric. This article summarises the results of different research projects realised by the authors over the last few years. They provide evidence that fragmentation not only leads to new patterns of social and ethnic segregation, and the redistribution of retail trade and amenities, but also contributes towards new forms of mobility and migration.

Keywords: urban development, globalisation, Chile, segregation, amenity migration

\section{Résumé: Le développement urbain fragmenté à} l'épreuve de la mondialisation: l'exemple de Santiago du Chili

La mondialisation a transformé la structure urbaine de toutes les villes d'Amérique latine. Le Chili fut le premier pays du sous-continent à entreprendre des réformes néolibérales dans les années 1970. Depuis cette période, le changement global, qui comprend à la fois le changement climatique et la mondialisation, a fortement influencé la capitale de Santiago du Chili. On peut notamment observer un processus de développement fragmenté à l'œuvre dans l'organisation urbaine contemporaine. Le présent article synthétise les résultats de plusieurs projets de recherche entrepris par les auteurs dans ce domaine au cours de ces dernières années. Ces résultats montrent que la fragmentation urbaine ne conduit pas seulement à une nouvelle forme de ségrégation sociale et ethnique et à une redistribution des centres du commerce de détail et des services, mais également à de nouvelles formes de mobilité et de migrations.

Mots-clés: développement urbain, mondialisation, Chili, ségrégation, migrations d'agrément

Prof. Dr. Axel Borsdorf, Universität Innsbruck, Institut für Geographie, Innrain 52, A-6020 Innsbruck, Österreich und Österreichische Akademie der Wissenschaften, Institut für Gebirgsforschung: Mensch \& Umwelt (IGF), ICT Technologiepark, Technikerstrasse 21a, A-6020 Innsbruck, Österreich.

e-mail: Axel.Borsdorf@uibk.ac.at

Prof. Dr. Rodrigo Hidalgo, Instituto de Geografia, Pontificia Universidad Católica de Chile, Av. Vicuña Mackenna 4860, Comuna de Macul, Santiago, Chile. e-mail: hidalgo@geo.puc.cl

\section{Manuskripteingang/received/manuscrit reçu le} 14.1.2011

Annahme zum Druck/accepted for publication/accepté pour publication: 24.6 .2011 